\title{
Beyond Chemoattraction: Multifunctionality of Chemokine Receptors in Leukocytes
}

\author{
Pilar López-Cotarelo ${ }^{1, *}$, Carolina Gómez-Moreira ${ }^{1} *$, Olga Criado-García ${ }^{1} *$, Lucas
} Sánchez ${ }^{2}$ and José Luis Rodríguez-Fernández ${ }^{1}$

${ }^{\mathbf{1}}$ Molecular Microbiology and Infection Biology Department. Centro de Investigaciones Biológicas. Consejo Superior de Investigaciones Científicas. Madrid. Spain

${ }^{2}$ Cellular and Molecular Biology Department. Centro de Investigaciones Biológicas. Consejo Superior de Investigaciones Científicas. Madrid. Spain

*Equal first authors

Chemokine is a combination of the words "chemotactic" and "cytokine", i.e. cytokines that promote chemotaxis. Hence, the term chemokine receptor refers largely to the ability to regulate chemoattraction. However, these receptors can modulate additional leukocyte functions, as exemplified by the case of CCR7 which, apart from chemotaxis, regulates survival, migratory speed, endocytosis, differentiation and cytoarchitecture. Here, we present evidence highlighting that multifunctionality is a common feature of chemokine receptors. Based on the activities that they regulate, we suggest that chemokine receptors can be classified in inflammatory (which control both inflammatory and homeostatic functions) and homeostatic families. The information accrued also suggests that the non-chemotactic functions controlled by chemokine receptors may contribute to optimize leukocyte functioning under normal physiological conditions and during inflammation.

"What's in a name? That which we call a rose

by any other name would smell as sweet." Romeo and Juliet (II, ii, 1-2)

Chemokine Receptors: What's in a Name? 
Chemokines constitute a large family of peptides that are characterized by their relatively high degree of similarity in their aminoacid sequences, small molecular weights and the presence of cysteines in conserved positions resulting in a characteristic 3Dstructure [1]. A common function for this family of proteins emerged in 1987, when it was found that interleukin-8 (IL-8, later renamed CXCL8), displayed potent chemoattractive activity for neutrophils, leading to the groundbreaking discovery that most members of this family of molecules promote directional migration [2]. In 1992, considering the high number of these ligands that were able to attract leukocytes, they were officially called chemokines, a combination of the words "chemotactic" and "cytokine", i.e. cytokines that promote chemotaxis (see Glossary). Studies on IL-8 showed very early that chemokines were able to regulate several functions in leukocytes in addition to chemotaxis, including respiratory burst, cell shape changes and exocytosis [3]. However, soon the field focused on chemotaxis, a function that is crucial for the control of the positioning of leukocytes during the immune response. In this regard, most work performed on chemokine receptors has focused on their ability to control chemotaxis. It could be that the name chemokine, which describes nicely a key function of these ligands, could have the unintended consequence of suggesting for non-specialists in the field of chemokines that these molecules controlled exclusively chemoattraction in leukocytes. Herein we present data indicating that chemokine receptors regulate multiple functions, implying that chemoattraction is only one of the several functions controlled by these receptors in leukocytes. We have also performed a functional classification of chemokine receptors based on the analysis of the specific functions that they control in leukocytes. This analysis suggest that chemokine receptors can be classified in inflammatory, which regulate both homeostatic and inflammatory functions, and homeostatic, which largely regulate homeostatic functions The list of functions compiled and the classification presented provides a useful framework to further our understanding of the critical roles played by chemokines and their receptors in the organization and execution of immune responses. Finally, together the data presented support the notion that multifunctionality of chemokine receptors may contribute to the efficient functioning of leukocytes in the immune system. 


\begin{tabular}{|c|c|c|}
\hline \multirow{2}{*}{ Receptor } & \multicolumn{2}{|c|}{ Leukocyte Expression } \\
\hline & Innate & Adaptive \\
\hline CXCR4 & Most innate leukocytes & Most adaptive leukocytes \\
\hline CXCR5 & & BC, Tfh, CD8+ Teff/mem, Tfr \\
\hline CCR6 & iDC, $\gamma \delta \mathrm{TC}, \mathrm{NK}, \mathrm{NKT}$ & Th17, Treg, Tfh \\
\hline CCR7 & $\mathrm{mDC}$ & Thymo, BCn, Tn, Tcm, Treg \\
\hline CCR8 & Mo-DC, Mo, МФ, $\gamma \delta$ TC & Th2, skin CD4rm, CD8 TC, Treg, thymo \\
\hline CCR9 & $\mathrm{DC}, \mathrm{pDC}$ & Thymo, gut-homing TC, BC \\
\hline XCR1 & Cross-presenting CD8+ DC, thymic DC & \\
\hline CXCR1 & Neu, Mo, NK, MC, Ba, DC, $\gamma \delta$ TC & T reg, CD8+ T eff, BC \\
\hline CXCR2 & Neu, Mo, NK, MC, Ba, DC, M $\Phi, \gamma \delta$ TC & CD8+ TC, Th17 \\
\hline CXCR3 & pDC, NK, NKT, Eos & BC, Th1, Treg, CD8+ Tcm and T eff/mem, Tfh \\
\hline CXCR6 & $\gamma \delta$ TC, NKT, NK, PC, ILC & Th1, Th17 \\
\hline CCR1 & Neu, Mo, MФ, Ba, DC & Th1, Tmem, pre-B \\
\hline CCR2 & $\mathrm{Mo}, \mathrm{M} \Phi, \mathrm{iDC}, \mathrm{Ba}, \mathrm{NK}$ & Th1 \\
\hline CCR3 & Eos, Ba, MC, MG, DC, PC & Th2 \\
\hline CCR4 & iDC, Mo, M $\Phi$ & $\begin{array}{l}\text { Th2, Th17, skin and lung- homing TC, Treg, BC, } \\
\text { CD4 and CD8 Teff/mem }\end{array}$ \\
\hline CCR5 & DC, Mo, MФ, NK, Neu & Th1, Th17, Treg, CD8+TC \\
\hline CX3CR1 & Mo, MФ, $\gamma / \delta$ TC, DC, NK, MG & Th1, CD8+ T eff/mem \\
\hline CCR10 & IgA+plasmablasts & Skin-homing TC \\
\hline
\end{tabular}

72

73 Table 1. Expression of chemokine receptors in leukocytes. The information on the 


\section{Current Classification of Inflammatory and Homeostatic Chemokines and their}

\section{Receptors in Leukocytes}

Chemokines have been classified into four families based on the relative position of the first conserved cysteine residues as they appear in the amino-terminal region of the peptide sequence [4]. These families are termed $\mathrm{XC}, \mathrm{CC}, \mathrm{CXC}$ and $\mathrm{CX} 3 \mathrm{C}$, respectively, where $\mathrm{C}$ represents a cysteine and $\mathrm{X}$ denotes any amino acid residue. Based on the physiologic context or type of expression that they present, chemokines can also be divided in three groups, namely, homeostatic (or constitutive), inflammatory (or inducible), and dual-type (homeostatic-inflammatory or constitutive-inducible) chemokines [7, 9]. Inflammatory chemokines (e.g. CXCL8) are induced in leukocytes and other cells in peripheral tissues in response to different threats to the organism, including microbial pathogens, irritants, or toxic cellular components. These chemokines are key players during the inflammatory response because they behave as "alarm signals" that attract specialized leukocytes which contribute to restore homeostasis [9]. Homeostatic chemokines (e.g. CXCL13) are constitutively expressed by resting cells and in this respect they regulate the migration and position of leukocytes at steady state. Finally, interestingly, dual-type (homeostaticinflammatory) chemokines (e.g. CXCL1, CCL21) can be homeostatic (constitutively expressed) in a certain context and inflammatory (inducible) in another context. Here a different context refers to organs, tissues or disease condition. For instance, the chemokine CXCL1 (receptor CXCR2) is induced (i.e. it is inflammatory) in response to infection in many peripheral tissues [10]; however, it is also constitutively expressed (i.e. it is homeostatic) in the bone marrow, where directs the homeostatic egress of neutrophils [11]. The chemokine CCL21 (receptor CCR7) is constitutively expressed (i.e. it is homeostatic) in the lymph nodes [12], attracting CCR7-expressing leukocytes (e.g. naïve T cells and dendritic cells) to these regions; however it is also induced (i.e. it is inflammatory) in chronic inflamed tissues, where it can also attract CCR7-expresing leukocytes [13, 14].

The effects of chemokines are mediated by chemokine receptors that belong to the Gprotein coupled receptor (GPCR) family (http://www.guidetopharmacology.org/ GRAC/FamilyDisplayForward?familyId=14). Emphasizing their important role in the immune system chemokine receptors are widely expressed in leukocytes involved in the innate and adaptive responses (Table 1). Chemokine receptors have been classified, according to the type of chemokine to which they bind in $\mathrm{XC}, \mathrm{CC}, \mathrm{CXC}$ or $\mathrm{CX} 3 \mathrm{C}$ classes (Table 2). Mirroring the classification of chemokines, chemokine receptors have also been 


\begin{tabular}{|c|c|c|c|}
\hline Ligand & Receptor & Leukocyte (function regulated) & Refs \\
\hline CXCL12 & CXCR4 & $\begin{array}{l}\text { CD4 TC ( } \alpha 4 \beta 1 \text { integrin activation, chemorepulsion, survival); } \\
\text { DC (Survival, chemorepulsion); Mo (Chemorepulsion, integrin } \\
\text { activation leading to arrest, differentiation); Neu } \\
\text { (Chemorepulsion); Tn (Integrin activation that enhances Tn-DC } \\
\text { adhesion and arrest, promotes Tr1 polarization); Thym } \\
\text { (Chemorepulsion); TC (Integrin activation leading to arrest, } \\
\text { enhanced T cell activation following IS formation); TC line } \\
\text { Jurkat (Integrin activation leading to enhanced adhesion to } \\
\text { antigen presenting cells) }\end{array}$ & [15-24] \\
\hline CXCL13 & CXCR5 & BC (Integrin activation leading to arrest) & {$[20,25]$} \\
\hline CXCL14 & Unknown & $\begin{array}{l}\text { CD14+ DC (Differentiation into Langerhans cells, survival); iDC } \\
\text { (differentiation) }\end{array}$ & {$[26,27]$} \\
\hline CCL20 & CCR6 & $\begin{array}{l}\text { BC (Integrin activation leading to adhesion); TC (Integrin } \\
\text { activation leading to arrest) }\end{array}$ & [20] \\
\hline CCL19 & CCR7 & $\begin{array}{l}\text { Ac Mo, Ac TC (increases expression of IL-10); BM-DC (Induces } \\
\text { proliferation); mDC (Enhances differentiation, survival, } \\
\text { endocytosis, chemokinesis, cytoarchitecture); NK (Co-stimulate } \\
\text { their proliferation); Tn (Survival, chemokinesis; LFA-1 integrin } \\
\text { activation leading to arrest); TC (Actin polymerization, } \\
\text { proliferation, survival) }\end{array}$ & $\begin{array}{l}{[12,20,} \\
24,28-47]\end{array}$ \\
\hline CCL21 & CCR7 & $\begin{array}{l}\text { BM-DC (Proliferation); mDC (Enhances differentiation, survival, } \\
\text { endocytosis, chemokinesis, cytoarchitecture); NK (Co-stimulate } \\
\text { their proliferation); Tn (Survival, chemokinesis; LFA-1 integrin } \\
\text { activation leading to arrest); TC (Actin polymerization, } \\
\text { proliferation) }\end{array}$ & $\begin{array}{l}{[12,20,} \\
24,28-45]\end{array}$ \\
\hline CCL1 & CCR8 & Thymoma (Survival) & [48] \\
\hline CCL18 & CCR8 & Th2 (LFA-1 integrin activation) & [49] \\
\hline CCL25 & CCR9 & TC line MT4C (Inhibits apoptosis induced by CD95) & [50] \\
\hline XCL1 & XCR1 & $\mathrm{CD8}^{+} \mathrm{DC}$ (Enhancement of the ability to activate CD8 TC) & [51] \\
\hline XCL2 & XCR1 & $\mathrm{CD8}^{+}$DC (Enhancement of the ability to activate CD8 TC) & [51] \\
\hline CXCL8 & CXCR1/CXCR2 & $\begin{array}{l}\text { MC (Chemokinesis); Neu (Activation, degranulation, respiratory } \\
\text { burst, release of gelatinase B, NET formation, chemorepulsion, } \\
\text { integrin activation leading to arrest, actin polymerization); Mo } \\
\text { (Respiratory burst, integrin activation leading to arrest); } \gamma \delta \text { TC } \\
\text { (Activation); pre-BC (Integrin activation leading to arrest); } \\
\text { CXCR1-transf Jurkat (Integrin activation leading to arrest) }\end{array}$ & $\begin{array}{l}{[3,20,52-} \\
60]\end{array}$ \\
\hline CXCL6 & CXCR1/CXCR2 & Neu (release of gelatinase B) & [61] \\
\hline CXCL7 & CXCR2 & $\begin{array}{l}\text { M1-MФ (Respiratory burst); Neu (Respiratory burst, } \\
\text { degranulation, elastase release, changes in cell morphology) }\end{array}$ & $\begin{array}{l}{[56,59,} \\
62,63]\end{array}$ \\
\hline CXCL5 & CXCR2 & 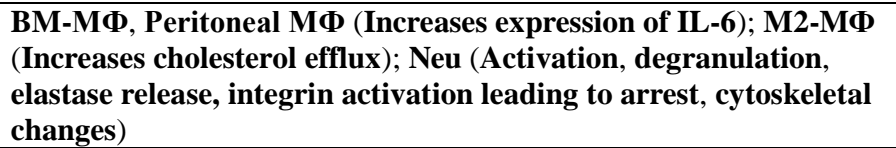 & $\begin{array}{l}{[20,63-} \\
65]\end{array}$ \\
\hline CXCL1 & CXCR2 & $\begin{array}{l}\text { Mo (Respiratory burst, integrin activation leading to arrest); Neu } \\
\text { (Respiratory burst, NET formation, degranulation, elastase } \\
\text { release, integrin activation leading to arrest, chemokinesis, actin } \\
\text { polymerization); TC (Th17 differentiation, IL-17 production) }\end{array}$ & $\begin{array}{l}{[11,20,} \\
56,60,63, \\
66-68]\end{array}$ \\
\hline CXCL2 & CXCR2 & Mo (integrin activation leading to arrest), Neu (chemokinesis) & $\begin{array}{l}{[11,20,} \\
66]\end{array}$ \\
\hline CXCL3 & CXCR2 & Mo (integrin activation leading to arrest) & [66] \\
\hline CXCL4 & CXCR3 & $\begin{array}{l}\text { Eos (Integrin activation and arrest); NK (CXCL8 secretion); TC } \\
\text { (Inhibits proliferation and IL-2 and Th1 cytokine release, } \\
\text { increases IL-4, IL-5, IL-13 cytokine release) }\end{array}$ & [69] \\
\hline CXCL4L & CXCR3 & No information available & \\
\hline CXCL10 & CXCR3 & CD4 TC (promotes Th1/Th17 polarization); pDCs & [70-74] \\
\hline
\end{tabular}




\begin{tabular}{|c|c|c|c|}
\hline & & $\begin{array}{l}\text { (Haptorepulsion); TC (Integrin activation and arrest, } \\
\text { proliferation); }\end{array}$ & \\
\hline CXCL9 & CXCR3 & $\begin{array}{l}\text { CD4 TC (promotes Th1/Th17 polarization); TC (Integrin } \\
\text { activation and arrest, proliferation) }\end{array}$ & {$[71-74]$} \\
\hline CXCL11 & CXCR3 & CD4 TC (Promotes proliferation, promotes Tr1 polarization) & $\begin{array}{l}{[71,72,} \\
74]\end{array}$ \\
\hline CXCL16 & CXCR6 & $\begin{array}{l}\text { TC (Adhesion), NKT (Survival, differentiation, increases } \\
\text { expression of cytokines IL-4 and IFN- } \gamma \text { ) }\end{array}$ & [75-77] \\
\hline CCL2 & CCR2 & $\begin{array}{l}\text { Ba (Histamine and Leukotriene C4 release); iDCs (inhibits IL-12 } \\
\text { expression); Mo (Enhances IL-1 and IL-6 production, } \\
\text { Arachidonic acid release, induces expression of integrins CD11c } \\
\text { and CD11b, integrin activation leading to arrest, inhibits IL-12); } \\
\text { MФ (Inhibits IL-12 expression, regulates lipid body biogenesis, } \\
\text { modulates differentiation); NK (Enhances cytolytic activity, } \\
\text { esterase release); TC (Survival, promotes Th2 proliferation) }\end{array}$ & $\begin{array}{l}{[20,38,} \\
78-85]\end{array}$ \\
\hline CCL13 & CCR1/CCR2/CCR3 & iDC, Mo (Inhibition of IL-12 production) & [78] \\
\hline CCL7 & $\begin{array}{c}\text { CCR1/CCR2/CCR3/ } \\
\text { CCR5 }\end{array}$ & iDC, Mo (Inhibition of IL-12 production) & [78] \\
\hline CCL8 & CCR1/CCR2 & iDCs, Mo ( inhibits IL-12 production) & [78] \\
\hline CCL4 & CCR5 & $\begin{array}{l}\text { CD8 }^{+} \text {DC (IL-12 synthesis induction); DC (Enhances } \\
\text { differentiation); Mo (Protease release, transendothelial } \\
\text { migration); NK (Cytolytic activity); TC (Transendothelial } \\
\text { migration, Th1 differentiation) }\end{array}$ & $\begin{array}{l}{[38,82,} \\
86,87]\end{array}$ \\
\hline CCL3 & CCR1/CCR5 & $\begin{array}{l}\text { Ac TC (promote IFN- } \gamma \text { production); CD8 }{ }^{+} \text {DC (IL-12 synthesis } \\
\text { induction); DC (Survival, enhances differentiation); Mo (Protease } \\
\text { release, transendothelial migration); Neu ( transendothelial } \\
\text { migration); NK (Enhances cytolytic activity); pre-BC (Integrin } \\
\text { activation leading to arrest); TC (Th1 differentiation, } \\
\text { transendothelial migration); CCR1-transf Jurkat (Integrin } \\
\text { activation leading to arrest) }\end{array}$ & $\begin{array}{l}{[20,38,} \\
82,84, \\
86-88]\end{array}$ \\
\hline CCL5 & $\begin{array}{c}\text { CCR1/CCR2/CCR3 } \\
\text { /CCR5 }\end{array}$ & 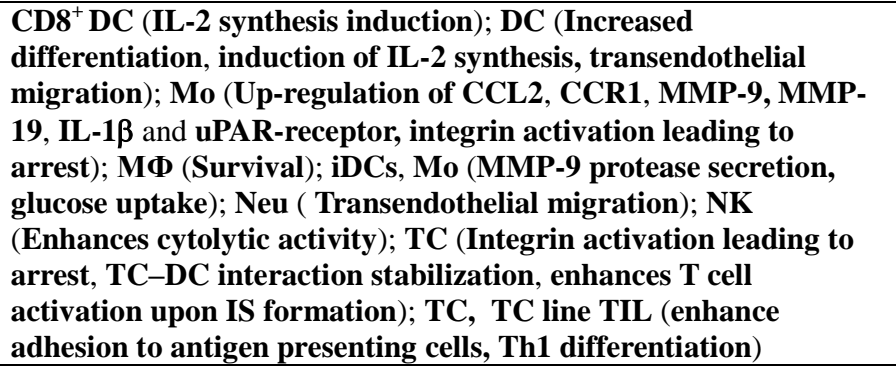 & $\begin{array}{l}{[15,20,} \\
38,86,87, \\
89,90]\end{array}$ \\
\hline CCL23 & CCR1 & $\begin{array}{l}\text { Mo cell line THP1 (Stimulates MMP-2 release and expression of } \\
\text { CCLL2, increase mRNA expression levels of TNF- } \alpha \text {, IL-1ß, CCL3, } \\
\text { ICAM-1, increased cell proliferation, L-selectin and CD11c); } \\
\text { Mo (Cytoskeletal remodeling, release of MMP-2) }\end{array}$ & [91-94] \\
\hline CCL14 & CCR1/CCR5 & No information available & \\
\hline CCL15 & CCR1/CCR3 & No information available & \\
\hline CCL16 & CCR1/CCR2/CCR5 & DC (Differentiation) & [95] \\
\hline CCL24 & CCR3 & No information available & \\
\hline CCL26 & CCR2/CCR3 & Eos (Respiratory burst, degranulation); Mo (Chemorepulsion) & {$[96,97]$} \\
\hline CCL11 & CCR3 & $\begin{array}{l}\text { Eos (Respiratory burst, Integrin activation leading to arrest, } \\
\text { actin polymerization) }\end{array}$ & {$[20,98]$} \\
\hline CCL17 & CCR4 & MФ (Differentiation) & [99] \\
\hline CCL22 & CCR4 & $\begin{array}{l}\text { MФ (Respiratory burst, phagocytosis, release of lysozomal } \\
\text { enzymes, exocytosis, differentiation,) }\end{array}$ & {$[99,100]$} \\
\hline CX3CL1 & CX3CR1 & $\begin{array}{l}\text { DC (Actin polymerization); Mo (Survival, integrin activation } \\
\text { leading to arrest); MФ (Survival); MG (Survival, phagocytosis); } \\
\text { NK (Activation, Enhances cytolytic activity, cytoskeletal } \\
\text { organization, integrin activation leading to arrest); } \\
\text { TC (Integrin activation leading to arrest) }\end{array}$ & $\begin{array}{l}{[20,38} \\
101-106]\end{array}$ \\
\hline CXCL17 & Unknown & No information available & \\
\hline CCL27 & CCR10 & No information available & \\
\hline CCL28 & CCR10 & No information available & \\
\hline
\end{tabular}




\section{Table 2. Non-chemotactic functions regulated by chemokine receptors in leukocytes.}

Homeostatic and inflammatory chemokines, chemokine receptors and the functions regulated by each receptor are indicated in green and red, respectively. Dual-type (homeostatic-inflammatory) chemokines are indicated in blue. Chemokine receptors on which no information is available are in black.

Abbreviations: Ac TC (Activated T cell); Ac Mo (Activated monocyte); Ba, basophil; BC (B cells), BCn, naïve B cell; BM-DC (Bone marrow-derived DCs); CD4rm (resident memory CD4 T cell); CD4 TC (CD4 helper T cell); CD8 TC (CD8 cytotoxic T cell); Eos (eosinophil); iDC (immature DC); mDC (mature DC); ILC (Innate lymphoid cell); $\boldsymbol{\gamma} \boldsymbol{\delta}$ TC $(\gamma \delta$ T cells), MC (Mast cell); MG (microglial cell); Mo (monocyte), MoDCs (monocyte-derived DCs); MФ (macrophage); Neu (neutrophil); NK (Natural killer cell); NKT (Natural killer $\mathrm{T}$ cell); M2-macrophages (M2-MФ); M1-macrophages (M1-MФ); PC (plasma cell); pDC (plasmacytoid DC); pre-BC (pre-B cells); P-MФ (Peritoneal macrophages); TC (T cell); Tcm (central memory T cell); Tfr (follicular regulatory T cell); Th1 (type 1 helper T cell); Th2 (type 2 helper T cell); Th17 (T helper 17 cells); Tn (naive T cell); Teff/mem (effector/memory T cell); Tfh (follicular helper T cell); Thymo (thymocytes); T reg (regulatory T cell); TIL (Tumor infiltrating lymphocytes); Tr1 (type 1 regulatory T cells), Tranf Jurkat (Transfected Jurkat cells); MT4C (MoltT 4 cells).

classified in inflammatory or homeostatic, depending on whether they bind to inflammatory or homeostatic chemokines. However, this classification is based only on the type of expression presented by the ligands. A functional classification of chemokine receptors requires an analysis of the specific functions controlled by these receptors in leukocytes and this has not been performed until now. A possible reason for this is that the studies on chemokine receptors have largely focused on chemotaxis, which is a key function of these receptors, and not in other functions. However, a functional classification can be useful to further our understanding of the role of these receptors during the immune response.

With the purpose of carrying out a functional classification of chemokine receptors that could be useful in future studies on the non-chemotactic functions of chemokine receptors, we have compiled in Table 2 all the information available on non-chemotactic functions controlled by chemokine receptors in different leukocytes. Most of the functional data presented in this table were obtained in in vitro studies. Note that only in the case of monogamous chemokine/chemokine receptor interactions the functional effects observed in vitro reflect the specific role of the receptor involved. Since for ligands that recognize more than one receptor, most published papers do not distinguish the role of a specific receptor, in these cases we include all the chemokine receptors potentially involved. 
151 Despite these drawbacks, the data gathered in the table provides important information on

152

153

154

155

156

157

158

159

160

161

162

163

164

165

166

167

168

169

170

171

172

173

174

175

176

177

178

179

180

181

182 the functionality of chemokine receptors in the immune system.

In view of the multiples activities of leukocytes controlled by chemokine receptors (Table 2), several functional classifications are possible. We have decided to classify these receptors based on their ability to control inflammatory and homeostatic functions because these two categories reflect the distinction between the execution and the organization of immune responses, respectively (see Box 1).

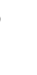

\section{Box 1. Inflammatory and Homeostatic Functions of Leukocytes}

Herein a leukocyte function is considered inflammatory when it is intrinsically associated to the inflammatory response, implying that it is active only during this response and that remains switched off during normal conditions. A leukocyte function is considered homeostatic when it is required under normal physiological conditions. Homeostatic functions are not necessarily shut off during inflammation because they may control basic processes of leukocytes.

Among the inflammatory functions are included respiratory burst [55, 56, 68, 97-100], NET formation [52, 53], phagocytosis (of pathogens), degranulation or exocytosis [3, 86]. Exocytosis is considered inflammatory or homeostatic depending on the functional outcome of the secretion. Secretion of the following pro-inflammatory components can be considered inflammatory: vasoactive amines [e.g. histamine [82]) and eicosanoids (e.g. leukotriene C4 [82]], which remodel the local vasculature in inflammatory sites; inflammatory cytokines [e.g. IL-1 [80, 89, 93], IL-6 [65, 80], IL-17 [68], TNF- $\alpha$ [93]) and chemokines (e.g. CXCL8 [69], CCL2 [89, 93], CCL3 [93]], which stimulate leukocytes during inflammation; and microbicide proteases [e.g. gelatinase [3], elastase [59, 82], and granzyme A [82]], which contribute to remove pathogens. Differentiation can be considered inflammatory when it occurs only during inflammation and the differentiated leukocyte promotes inflammatory responses at inflammatory sites. For instance, dendritic cells promote differentiation of naïve CD4 T cells in effector Th1 [71-73, 84] or Th17 [68] CD4 $\mathrm{T}$ cells, which migrate to inflammatory sites where they secrete pro-inflammatory cytokines. Hence, Th1 and Th17 differentiation from CD4 T cells can be considered an inflammatory process. 
183

184

185

186

187

188

189

190

191

192

193

194

195

196

197

198

199

200

201

202

203

204

205

206

207

208

209

210

211

212

213

214

215

216

Among the homeostatic functions are included survival [19, 26, 31, 33, 34, 39, 48, 50, 77], proliferation [92] and endocytosis [44, 102, 104-106], multiple processes associated with leukocyte migration, including actin polymerization and cytoskeletal remodeling [42, 43], integrin activation resulting in adhesion to the extracellular matrix (arrest) or other cells (cell-cell binding) [20], chemotaxis, haptotaxis and chemokinesis [37], transendothelial migration [82], chemorepulsion [17], haptorepulsion [70]. The secretion of proteins that regulate homeostatic functions or that do not directly promote, or even inhibit inflammation can be considered homeostatic. For instance, the secretion of CCL19, which induces proliferation in T cells, is homeostatic [36]. The release of antiinflammatory IL-10 by monocytes, upon stimulation by CCL19, can be considered also homeostatic [107]. Differentiation can be considered homeostatic when it takes place under normal homeostatic conditions and the differentiated leukocyte does not directly promote inflammation in inflammatory sites [21, 26]. For instance, monocyte-macrophage [21] and precursor-Langerhans cell [26] differentiation induced by CXCL12 and CXCL14, respectively, can be considered homeostatic.

\section{Classifying Chemokine Receptors According to Inflammatory and Homeostatic} Immune Functions

To classify chemokine receptors according to their capability to control homeostatic or inflammatory functions, as defined in Box 1, the strategy that we used is the following. First, in Table 2 we divided the functions controlled by each chemokine receptor in inflammatory (in red) and homeostatic (in green). Second, chemokine receptors that control both inflammatory and homeostatic functions were considered inflammatory (in red), and receptors that regulate exclusively homeostatic functions were considered homeostatic (in green). To classify a certain receptor as inflammatory, it was sufficient that at least one of its ligands was able to control one inflammatory function. Eg. CCR3 is considered inflammatory because its ligand CCL11 induces a respiratory burst in eosinophils, even though there is no information on the functional effects of the ligand CCL24.

It is important to note here that in the case of chemokines the names inflammatory and homeostatic can be misleading. They do not refer to the function, but they just describe the context (homeostasis or inflammation) where they are expressed or the type of expression (constitutive or inducible) that they present. However, interestingly, once receptors are 
217

218

219

220

221

222

223

224

225

226

227

matched with their ligands in Table $\mathbf{2}$, we observed that inflammatory and homeostatic chemokines recognized receptors that we had classified as inflammatory and homeostatic, respectively. Therefore, our analysis also suggests that the context/type of expression (inflammatory/homeostatic or inducible/constitutive) of a chemokine predicts the functional category of its respective receptor. Granting this, however, only a functional analysis provides a precise functional definition of a chemokine receptor. For instance, only this type of analysis can show that inflammatory receptors control both homeostatic and inflammatory functions or that all dual-type chemokine receptors are functionally either inflammatory or homeostatic (see below).

Our analysis also showed that receptors for dual-type chemokines (homeostaticinflammatory) can fall in either group, providing a new rational approach to subset these chemokine-receptor pairs. The data presented in Table 2 indicate that the group including CCR3 (dual ligand CCL11) [108, 109], CCR4 (dual ligands CCL17, 22) [110, 111], CXCR2 (dual ligands CXCL1,2) [11], CXCR3 (dual ligands CXCR9, 10, 11) [112] and CX3CR1 (dual ligand CX3CL1) [113] regulate both inflammatory and homeostatic functions, and therefore can be classified as inflammatory (Table 2 and Fig 1). On the other hand, the group encompassing the receptors CCR6 (dual ligand CCL20) [114], CXCR6 (dual ligand CXCL16) [115], XCR1 (dual ligand XCL1) [51, 116, 117], CXCR4 (dual ligand CXCL12) [118], CCR7 (dual ligand CCL19 and CCL21) [13, 14, 119], CCR9 (dual ligand CCL25) [114, 120], CXCR5 (dual ligand CXCL13) [119] and CCR8 (dual ligand CCL1) [121] control largely homeostatic functions and hence can be classified as homeostatic. In summary, based on the analysis of the functions controlled by the chemokine receptors in leukocytes, all chemokine receptors can be divided in inflammatory, which control inflammatory and homeostatic functions, and homeostatic, which control largely homeostatic functions (Fig. 1 and Table 2). The division based on the functions controlled by chemokine receptors in leukocytes allows categorizing all chemokine receptors independently of the type ligand that they bind, avoiding the ambiguity that emerges when a receptor has dual-type ligands. The analysis of the functions of the receptors for dual-type chemokines also suggests that chemokine receptors have an intrinsic inflammatory or homeostatic nature that does not change when they are exceptionally exposed to an alternative context. This is especially clear in the case of homeostatic receptors that seem to keep their homeostatic functions even when their ligands are expressed during chronic inflammation (e.g. CCR7 and its dual-context ligands CCL19 and CCL21 [13, 14, 119] or CXCR4 and its dual ligand CXCL12 [118]). Finally, it 
251 is important to indicate that although the classification of chemokine receptors presented in

252 Table 2 is useful, it has to be necessarily considered provisional until the whole set of

253 functions controlled by these receptors is completed.

254

255

256

257

258

259

260

261

262

263

264

265

266

267

268

269

270

271

272

273

274

275

276

277

\section{Impact of Chemokine Receptor Signaling on Immune Functions}

\section{Chemokine receptors control rapid functional responses}

Chemokine receptors control a variety of rapid functional responses that do not require changes in gene expression. Below, we analyze first rapid functional responses controlled by inflammatory receptors and second by homeostatic receptors. As inflammatory receptors control inflammatory and homeostatic functions, below we analyze both types of responses. Rapid inflammatory functions controlled by inflammatory receptors are particularly important in innate leukocytes during inflammation because these cells should respond swiftly to the threats to the organism (Table 2). Neutrophils are the first innate leukocytes that migrate to inflammatory sites, followed by monocytes. In these sites can be also found macrophages, immature dendritic cells and NK cells. Eosinophils are present during helminth parasitic infections and allergic diseases. As shown in Tables $\mathbf{1}$ and 2, all these leukocytes express inflammatory chemokine receptors that control rapid inflammatory responses. In this regard, CXCR1/CXCR2 control in neutrophils respiratory burst [3, 56, 59, 63, 68], NET formation [52, 53], degranulation [3, 59] and bactericidal protease release [61]. CXCR1/CXCR2 also modulates in monocytes respiratory burst [56]. Bactericidal protease release can be also regulated by CCL5 (receptors CCR1/CCR2/CCR3/CCR5) in dendritic cells [90], by CCR5 in monocytes, neutrophils and NK cells [82] and by CCR1 in monocytes [89] and by CCR4 in macrophages [99, 100]. CCR4 controls respiratory burst in macrophages [99, 100]. CCR2/CCR3 control respiratory burst and degranulation in eosinophils [96, 97]. Finally, in NK cells CCR2, CCR1/CCR5 and CX3CR1 [38] regulate the cytolytic activity and CCR2 [122] controls bactericide protease release. Inflammatory receptors also control rapid homeostatic function in innate and adaptive leukocytes. Integrin-mediated adhesion can be controlled by CXCR1/CXCR2 in monocytes and neutrophils [20, 66], by CXCR3 in eosinophils and T cells [20], by CCR2 in monocytes [20], by CCR3 in eosinophils [20], and by CCR1 and CXCR1 in transfected Jurkat cells [20]. Integrin-mediated adhesion is also involved in transendothelial migration of leukocytes from the vessels to the tissues where it is located the inflammatory focus. Cytoarchitecture can be controlled by the inflammatory receptor CXCR1/CXCR2 in neutrophils [11], by CCR1 in monocytes [94], by CCR3 in eosinophils 
285 [59], by CX3CR1 in NK cells [103]._Chemokinesis can be controlled by the inflammatory

286

287

288

289

290

291

292

293

294

295

296

297

298

299

300

301

302

303

304

305

306

307

308

309

310

311

312

313

314

315

316

317

318 receptor CXCR1/CXCR2 in neutrophils [11] and mast cells [55].

Regarding the homeostatic receptors, as indicate above, they control homeostatic functions. Below we discuss rapid homeostatic functions controlled by these receptors in innate and adaptive leukocytes. Integrin-mediated adhesion is necessary to promote leukocyte adhesion and movement on the 2D surfaces of the capillary vessels and during transendothelial migration from the vessels to the tissues during the homeostatic migration of leukocytes. Integrin-mediated adhesion is controlled by CCR6 and CXCR5 in B cells $[20,25]$, by CXCR4 in T cells [20], by CCR7 in lymphocytes [20, 29, 30] and by CCR8 in T cells [49]. The homeostatic receptor CCR7 control cytoarchitecture [42, 43], endocytosis [44] and chemokinesis in dendritic cells [37]. In summary, the data suggest that inflammatory receptors control in innate leukocytes rapid functional responses that contribute to combat effectively pathogens and other threats to the organism; in addition, these receptors also drive homeostatic functions that modulate dynamic migratory processes of leukocytes in the immune system. On the other hand, homeostatic receptors control a variety of rapid homeostatic functions, including migration or endocytosis, that allow efficient functioning of innate and adaptive leukocytes in the immune system.

\section{Signaling Pathways Involved in Chemokine Receptor-controlled Rapid Functional}

\section{Responses}

Although it is well known that chemokine receptors control multiple signaling pathways in leukocytes, most studies have focused on the signaling molecules driving chemotaxis $[12,123]$. From the studies available it emerges that the $\mathrm{Gi}$ family of $\mathrm{G}$ proteins plays a key role in the regulation of most functions controlled by chemokine receptors $[37,39,43$, 44]. Moreover, at least in the case of chemotaxis, the $G_{\beta \gamma}$ subunits seem to play a predominant role, compared to the $\mathrm{G}_{\alpha \mathrm{i}}$ subunits $[37,39,124]$. In addition to Gi proteins, $\mathrm{G}_{12 / 13}$ molecules may also play important roles in the regulation of actin-actin dependent functions like cytoarchitecture and endocytosis [37, 42]. Downstream of G proteins, serinethreonine kinases like MAPKs, Akt or tyrosine kinases like JAK, Src and Pyk2 have been involved in the regulation of chemotaxis, migration and adhesion [37, 41, 42, 92, 123]. Chemokine-receptor regulated actin dynamics involve small GTPases, including Rho, Rac or Cdc42 and their downstream signaling molecules, including the serine-threonine kinases ROCK, LIMK and its targets cofilin or the myosin light chain kinase [37, 42-44]. Interestingly, CCR7 uses relatively independent signaling modules to control its different 
319

320

321

322

323

324

325

326

327

328

329

330

331

332

333

334

335

336

337

338

339

340

341

342

343

344

345

346

347

348

349

350

351

functions $[12,31,34,37,39,42]$. However, it is not known how common is this type of segregation of the signaling in modules, although clearly it is not a general mechanism because, for instance, it is not observed in the case of CXCR4 [19]. In summary, additional efforts are required to get a clear picture on the mechanisms used by chemokine receptors to regulate its short term functions.

\section{Chemokine Receptors Control Long-term Functional Responses}

In addition to short term responses, chemokine receptors (inflammatory and homeostatic) may also regulate long term responses, involving changes in gene expression that can shape the environment of leukocytes (Table 2). Inflammatory receptors regulate for instance leukocyte differentiation (CXCR2, CXCR6, CCR1/CCR5, CCR1/CCR2/CCR3/CCR5), proliferation (CXCR3, CCR1/CCR5), cytokine expression (CXCR3) and survival (CCR2, CCR1/CCR5, CCR1/CCR2/CCR3/CCR5, CX3CR1). The chemokine CCL5 (receptors CCR1/CCR3/CCR5) induces in monocytes up-regulation of cytokine IL-1 $\beta$ and the chemokines CCL2, CCL3, CCL4, and CXCL8. CCR1 induces upregulation of metalloproteinases [89]. Homeostatic receptors regulate survival (CXCR4, CXCR5, CCR7, CCR9 and the unknown receptor of CXCL14), differentiation (CCR7, and the CXCL14 receptor) and proliferation (CCR7 and CCR8).

Signaling Pathways Involved in Chemokine Receptor-controlled Long-term Functional Responses

Since long-term functions involve expression of new genes, transcription factors are often involved in these processes. In this regard, it has been shown that chemokine receptors can regulate multiple transcriptional factors, including STATs (Signals Transducer and Activators of Transcription) [125], NFкB (Nuclear factor kappa-lightchain-enhancer of activated B cells) [39], CREB (cAMP response element-binding) protein [126], NFAT (Nuclear factor of activated T-cells), FOXO1/3 (forkhead box O (FoxO) transcription factors 1/3) [19, 31] and AP-1 (Activation Protein-1) [126], among other factors. Albeit the information available on the role of transcription factors in the regulation of long-term functions in leukocytes is sparse, the examples available support that they may modulate the long-term effects of chemokines. CCL3 (receptors CCR1 and CCR5) regulates myeloid gene expression and differentiation through the control of AP-1 and CREB [126]. CCR7 regulates survival in dendritic cells through the activation of the 
352

353

354

355

356

357

358

359

360

361

362

363

364

365

366

367

368

369

370

371

372

373

374

375

376

377

378

379

380

381

382

383

384

385

$\mathrm{NF \kappa B}$, which controls the pro-survival Bcl2 family member $\mathrm{Bcl}_{\mathrm{xl}}$. CCR7 also promotes survival by inducing inhibition of FOXO1/3, which controls the pro-apoptotic Bcl2 family member Bim [31, 39]. CCR5 drives T cell proliferation by inducing stimulation of STATs [125]. CXCL10 (receptor CXCR3) promotes Th1 polarization via STAT1, STAT4, and STAT5 [74]. CXCR4 promotes survival in dendritic cells through inhibition of the axis FOXO1/3-Bim [19].

\section{The Effects of Chemokine Receptors in Leukocyte Functions are Dynamic}

Leukocytes are largely migratory cells that are exposed to a variety of chemokines in different tissues. They can also undergo differentiation and changes the expression of chemokine receptors during their life cycle. This implies that chemokine receptors exert a dynamic regulation of the functions of leukocytes in the immune system. Although there are multiple examples, this concept can be illustrated in the case of dendritic cells (DCs) [127]. In peripheral tissues DCs are found in a differentiation stage called immature (immature DCs, iDC), in which they express the inflammatory chemokine receptors CXCR2, CCR1, CCR2, CCR3, CCR5 and CCR6 and the homeostatic receptor CXCR4 (Table 2). Upon exposure to pathogens or other danger signals, iDCs undergo a differentiation program and become mature DCs (mDCs). The process results in the dampening of the expression of the inflammatory receptors and up-regulation of the homeostatic receptor CCR7, while CXCR4 does not change substantially its levels. The increase in the expression of CCR7 promotes the migration of mDCs through the afferent lymphatics to the lymph nodes (LNs), attracted by the ligands CCL21, expressed in the afferent lymphatics, and by CCL19 and CCL21 expressed in the lymph nodes. CXCR4 also contributes to direct the mDCs to the LNs [19]. Hence, upon maturation the functions of the mDCs can be modulated by the homeostatic receptors CCR7 and CXCR4 (see next section) (Table 2).

\section{Homeostatic and Inflammatory Chemokine Receptors in the Immune system}

The results presented suggest that under normal physiologic conditions homeostatic chemokine receptors drive homeostatic activities that contribute to the optimal functioning of innate and adaptive leukocytes in the immune system. Moreover, under conditions in which the organism faces pathogens or other threats, inflammatory receptors can be important components of the inflammatory response because, in addition to homeostatic functions, they control inflammatory responses that confer leucocytes a higher capability to 
386

387

388

389

390

391

392

393

394

395

396

397

398

399

400

401

402

403

404

405

406

407

408

409

410

411

412

413

414

415

416

417

418

419

combat threats and defend the organism. Below we provide some examples that illustrate these concepts. First we provide examples of homeostatic receptors expressed by leukocytes in homeostatic settings. In the case of the mature DCs (mDCs), commented in the prior section, CCR7 controls additional functions apart from controlling the migration of these cells to the lymph nodes. In this regard, CCR7 enhances the differentiation of the mDCs, increasing their ability to activate $\mathrm{T}$ cells in the $\mathrm{LN}$ [128]. It also induces an increase in the migratory speed $[37,129]$, contributing to increase the number of mDCs that access lymph nodes [12, 37, 39, 129]. CCR7 signaling contributes to maintain the characteristic dendritic morphology, with an extended cell surface, which favors the number of contacts with $\mathrm{T}$ cells during antigen presentation [43]. CCR7-induced endocytosis increases the ability of mDCs to incorporate antigens originating in tissues as they advance through the afferent lymphatics or when they are positioned in the LNs, where T cell activation takes place $[44,130]$. Both CCR7 and CXCR4 induce survival of mDCs, which increase the number of these leukocytes that reach the LNs [19, 39]. Furthermore, CXCR4, CXCR6, CCR6, CCR7 and CCR8 control integrin activation leading to arrest in $\mathrm{T}$ cells. This suggests that under normal physiologic conditions CXCR4, CXCR6, CCR6 and CCR7 play an important role in the control of the process of adhesion and extravasation of leukocytes from blood vessels.

In the presence of pathogens or other threats a variety of innate leukocytes represent the first line of defense of the organism. Inflammatory receptors regulate in innate leukocytes key inflammatory functions that contribute to fend off these threats. Neutrophils are the first innate leukocytes that migrate to the inflamed regions from blood vessels. The inflammatory receptors $\mathrm{CXCR} 1$ and $\mathrm{CXCR} 2$ promote in neutrophils inflammatory activities involved in pathogen destruction, including respiratory burst, degranulation leading to the liberation of proteases and NET formation, [52, 53, 55, 59, 61, 63, 64]. In addition, CXCR1 and CXCR2 also control in neutrophil homeostatic activities, including chemokinesis, integrin activation and adhesion to the vascular endothelium, some of which are required for the extravasation to inflamed tissues [11,20]. Monocytes are innate immune cells that may reside in peripheral tissues. In monocytes CXCR1 and CXCR2 promote respiratory burst, contributing to pathogen destruction [56]. CXCR1 and CXCR2 also regulate in monocyte homeostatic functions, including integrin activation leading to arrest and adhesion to the endothelium, facilitating the extravasation of monocytes from blood vessels to inflamed sites [20,131]. Macrophages are highly phagocytic cells that efficiently remove pathogens in inflamed tissues. The inflammatory receptor CCR4 
promotes in macrophages respiratory burst and phagocytosis, both activities contribute to the killing and clearance of pathogens [100]. In addition, CCR4 controls a homeostatic function such as M1/M2 macrophage differentiation [99]. CXCR2 controls in macrophages respiratory burst and, consequently, pathogen destruction [62]. Natural Killer (NK) cells recognize and kill infected or damaged cells and cancer cells. NK cells secrete perforin, a membrane-disrupting protein, and granzymes, to selectively kill target cells. CX3CR1 controls NK cells activation, an inflammatory function, and in addition homeostatic activities like integrin activation leading to arrest and cytoskeletal organization, functions related to the migratory properties of these cells or their ability to extravasate to inflamed tissues [20, 103]. Finally, it should be noted that during inflammation chemokines act in concert through multiple chemokine receptors to control several leukocyte types, suggesting that altogether the non-chemotactic functions of these receptors could have an appreciable impact on the immune response.

The examples presented above on the potential functional effect of chemokine receptors in vivo were largely inferred from available data obtained from in vitro studies (Table 2). The interpretation of in vivo data on the functions of leukocytes regulated by chemokine receptors, for instance, experiments with knock-out mice, is difficult, unless prior functional information obtained in vitro is available. Experiments in which a chemokine receptor may be conditionally switched-off, may help to define the function/s of a receptor expressed by leukocyte in a specific niche in vivo. Until these genetic studies become available, when performing in vivo studies, it is advisable to carry out a parallel in vitro analysis to get insight into the functions that a chemokine receptor may control in the leukocyte involved in the response obtained. In this regard Table 2 may be useful in future studies.

\section{Concluding remarks}

Chemokine receptors are widely expressed in leukocytes involved in the innate and adaptive arms of the immune system. Studies on chemokine receptors have largely focused on their chemoattractive capabilities; however, the data accrued during the last years indicate that they also control multiple leukocyte functions. In this regard, the information gathered in this review indicates that chemokines are not just directional signals. They are also cues that convey information to leukocytes on the environment (homeostatic or inflammatory) and, through chemokine receptors, modulate their behavior and consequently the immune response. Until now the definition of inflammatory or 
454

455

456

457

458

459

460

461

462

463

464

465

466

467

468

469

470

471

472

473

474

475

476

477

478

479

480

481

482

483

484

485

486

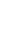

homeostatic chemokine receptor was only descriptive because it only indicated that a receptor was able to recognize inflammatory or a homeostatic chemokines. Based on the functional information obtained, we classify these receptors in inflammatory, which regulate inflammatory and homeostatic functions, and homeostatic, which only control homeostatic functions. This classification provides a clearer functional description of chemokine receptors that can be valuable in future analysis of these receptors.

Many questions will have to be addressed in the future studies to gain a better understanding of the non-chemotactic functions of chemokine receptors in leukocytes (see Outstanding Questions). Until now most in vivo studies on chemokine receptors and, in particular, work analyzing the phenotypical effects of the deficiency of these receptors in animal models, have interpreted the results obtained considering that they controlled mostly chemotaxis (e.g. [73, 132-136]). However, it is possible that non-chemotactic functions controlled by these receptors may have contributed at least partially to the effects observed in the in vivo studies. In this regard, it is important to carry out in the future functional studies to get a more complete understanding of the roles of these receptors during the immune response. Chemokine receptors play important roles in the immune system under normal physiological conditions and in multiple chronic inflammatory diseases [137]. Getting insight into the non-chemotactic functions performed by these receptors and the mechanisms involved may facilitate the development of pharmacological strategies to modulate the function of the leukocytes involved in these processes, to induce more robust immune responses under normal conditions or to dampen these responses during chronic inflammation

\section{Acknowledgements}

JLRF was supported by grants awarded by Ministerio de Ciencia e Innovación (SAF200801468), Ministerio de Economía y Competitividad (SAF2011-23890, SAF2014-53151-R), RIER (RETICS Program / Instituto de Salud Carlos III) (RD08/0075) and Consejería de Educación y Empleo from Comunidad de Madrid (Raphyme, S2010/BMD-2350). PLC was supported by an FPI scholarship (Ministerio de Economía y competitividad). C.G.-M. was supported by a contract associated with Grant Raphyme S2010/BMD-2350 (Consejería de Educación y Empleo, Comunidad de Madrid). 
509 Figure 1. All chemokine receptors, including receptors of dual-type chemokines, can

510 be classified as homeostatic or inflammatory. The study of the function controlled by

511 chemokine receptors in leukocytes indicates that they all, including the receptors dual-

512 type chemokines, can be functionally divided in inflammatory receptors, which control

513 homeostatic and inflammatory functions, and homeostatic receptors, which regulate 514 largely homeostatic functions.

515

\section{Glossary}

517 Arrest: Integrin-mediated process whereby circulating leukocytes rolling in vascular 518 beds slow down and stop prior to their migration to inflammatory sites or lymph nodes.

519 Actin polymerization: Process whereby a filament of actin incorporates new 520 monomers of actin which cause the growth of the filament. 
521 Chemoattraction: directional motility of a cell in response to a gradient of soluble or

522 bound-to the substrate chemoattractant, hence encompasses chemotaxis and haptotaxis.

523 Chemokinesis (or increase in migratory speed): increased non-directional speed of a 524 cell in response to a chemokine.

525 Chemorepulsion (or fugotaxis): directional migration of a cell away from a gradient of 526 soluble chemokine.

527 Chemotaxis: directional motility of a cell in response to a gradient of soluble 528 chemoattractant.

529 Cytoskeletal remodeling: reorganization of the cytoskeletal components of a cell 530 leading to changes in cell shape.

531 Degranulation: exocytic process whereby vesicles called granules (typical of 532 neutrophils, mast cells, basophils and eosinophils) release their content to the 533 extracellular media.

534 Exocytosis: process whereby secretory vesicles encapsulating different secretory 535 products (proteases, cytokines, chemokines hormones and other cellular products) are 536 exported out of the cell. The vesicles fuse with the plasma membrane and release their 537 content to the extracellular space.

538 Haptorepulsion: directional movement of a cell away from a substrate-bound 539 chemoattractant.

540 Haptotaxis: directional motility of a cell in response to a gradient of a substrate-bound 541 chemoattractant.

542 Integrin activation: molecular process whereby integrin molecules undergone changes 543 in structure (changes in affinity) and/or aggregation on the membrane (clustering) 544 resulting in the acquisition of the ability to bind to their ligand/s.

545 Migration: name given to the translation of cells from one location to another. Note 546 that although often used as a synonymous of chemoattraction it is not the same.

547 Neutrophil extracellular trap (NET): network of extracellular fibers composed of 548 deoxyribonucleic acid (DNA), histones, and antimicrobial granule proteins released 549 from neutrophils. NETs snare and kill invasive bacteria.

550 Phagocytosis: process whereby specialized cells engulf large particles, including 551 apoptotic cells, virus or bacteria. After phagocytosis the particles are encapsulated in 552 large vacuoles called phagosomes, which fuse with the lysosomes, resulting in the 553 destruction of the particles by free radicals and proteases.

554 Respiratory burst (or oxidative burst): rapid release from cells of reactive oxygen 555 species (ROS), including superoxide radical and hydrogen peroxide. ROS is generated 556 by the membrane-bound enzyme nicotinamide adenine dinucleotide phosphate-oxidase 557 (NADPH). The ROS generated possess microbicidal functions. 
558 Transendothelial migration (or diapedesis): migration of leukocytes between two 559 endothelial cells to reach inflammatory sites or the lymph nodes. It is a complex 560 function that involves at least chemoattraction, adhesion and motility.

REFERENCES

579 1. Fernandez, E.J. and Lolis, E. (2002) Structure, function, and inhibition of chemokines. Annu 580 Rev Pharmacol Toxicol 42, 469-499.

581 2. Yoshimura, T. et al. (1987) Purification of a human monocyte-derived neutrophil 582 chemotactic factor that has peptide sequence similarity to other host defense cytokines. Proc $583 \quad$ Natl Acad Sci U S A 84, 9233-9237.

584 3. Baggiolini, M. et al. (1989) Neutrophil-activating peptide-1/interleukin 8, a novel cytokine 585 that activates neutrophils. J Clin Invest 84, 1045-1049.

586 4. Bachelerie, F. et al. (2014) International Union of Basic and Clinical Pharmacology. LXXXIX. 587 Update on the extended family of chemokine receptors and introducing a new nomenclature 588 for atypical chemokine receptors. Pharmacol Rev 66, 1-79.

589 5. Griffith, J.W. et al. (2014) Chemokines and chemokine receptors: positioning cells for host 590 defense and immunity. Annu Rev Immunol 32, 659-702.

591 6. Viola, A. and Luster, A.D. (2008) Chemokines and their receptors: drug targets in immunity and inflammation. Annu Rev Pharmacol Toxicol 48, 171-197.

593 7. Zlotnik, A. and Yoshie, O. (2012) The chemokine superfamily revisited. Immunity 36 (5), 70559416.

595 8. Olson, T.S. and Ley, K. (2002) Chemokines and chemokine receptors in leukocyte trafficking. 596 Am J Physiol Regulatory Integrative Comp Physiol 283, R7-R28. 
9. Moser, B. et al. (2004) Chemokines: multiple levels of leukocyte migration control. Trends Immunol 25, 75-84.

10. Wengner, A.M. et al. (2008) The coordinated action of G-CSF and ELR + CXC chemokines in neutrophil mobilization during acute inflammation. Blood 111, 42-49.

11. Kohler, A. et al. (2011) G-CSF-mediated thrombopoietin release triggers neutrophil motility and mobilization from bone marrow via induction of Cxcr2 ligands. Blood 117, 4349-4357.

12. Sanchez-Sanchez, N. et al. (2006) The multiple personalities of the chemokine receptor CCR7 in dendritic cells. J. Immunol. 176, 5153-5159.

13. Johnson, L.A. and Jackson, D.G. (2010) Inflammation-induced secretion of CCL21 in lymphatic endothelium is a key regulator of integrin-mediated dendritic cell transmigration. Int Immunol 22 (10), 839-49.

14. Grant, A.J. et al. (2002) Hepatic expression of secondary lymphoid chemokine (CCL21) promotes the development of portal-associated lymphoid tissue in chronic inflammatory liver disease. Am J Pathol 160 (4), 1445-55.

15. Molon, B. et al. (2005) T cell costimulation by chemokine receptors. Nat Immunol 6, 465471.

16. Huttenlocher, A. and Poznansky, M.C. (2008) Reverse leukocyte migration can be attractive or repulsive. Trends Cell Biol. 18, 298-306.

17. Poznansky, M.C. et al. (2000) Active movement of T cells away from a chemokine. Nat Med 6, 543-548.

18. Cascio, G. et al. (2015) CXCL12 Regulates through JAK1 and JAK2 Formation of Productive Immunological Synapses. J Immunol 194, 5509-5519.

19. Delgado-Martin, C. et al. (2011) Chemokine CXCL12 uses CXCR4 and a signaling core formed by bifunctional Akt, extracellular signal-regulated kinase (ERK)1/2, and mammalian target of rapamycin complex 1 (mTORC1) proteins to control chemotaxis and survival simultaneously in mature dendritic cells. J Biol Chem 286, 37222-37236.

20. Ley, K. (2003) Arrest chemokines. Microcirculation 10, 289-95.

21. Sanchez-Martin, L. et al. (2011) The chemokine CXCL12 regulates monocyte-macrophage differentiation and RUNX3 expression. Blood 117, 88-97.

22. Kabashima, K. et al. (2007) CXCR4 engagement promotes dendritic cell survival and maturation. Biochem Biophys Res Commun 361, 1012-1016.

23. Suzuki, Y. et al. (2001) Diverse transcriptional response of CD4+ T cells to stromal cellderived factor SDF-1: cell survival promotion and priming effects of SDF-1 on CD4+ T cells. J Immunol 167 (6), 3064-3073.

24. Kaiser, A. et al. (2005) CC chemokine ligand 19 secreted by mature dendritic cells increases naive $T$ cell scanning behavior and their response to rare cognate antigen. J Immunol 175, 2349-56.

25. Kanemitsu, N. et al. (2005) CXCL13 is an arrest chemokine for B cells in high endothelial venules. Blood 106 (8), 2613-8.

26. Schaerli, P. et al. (2005) Cutaneous CXCL14 targets blood precursors to epidermal niches for Langerhans cell differentiation. Immunity 23, 331-342.

27. Shurin, G.V. et al. (2005) Loss of new chemokine CXCL14 in tumor tissue is associated with low infiltration by dendritic cells (DC), while restoration of human CXCL14 expression in tumor cells causes attraction of DC both in vitro and in vivo. J Immunol 174 (9), 5490-8.

28. Bardi, G. et al. (2003) Rho kinase is required for CCR7-mediated polarization and chemotaxis of T lymphocytes. FEBS Lett $542,79-83$.

29. Campbell, J.J. et al. (1998) Chemokines and the arrest of lymphocytes rolling under flow conditions. Science 279 (5349), 381-4.

30. Constantin, G. et al. (2000) Chemokines trigger immediate beta2 integrin affinity and mobility changes: differential regulation and roles in lymphocyte arrest under flow. Immunity $13(6), 759-69$. 
31. Escribano, C. et al. (2009) CCR7-dependent stimulation of survival in dendritic cells involves inhibition of GSK3beta. J Immunol 183 (10), 6282-95.

650 32. Flanagan, K. et al. (2004) The lymphoid chemokine CCL21 costimulates naive T cell expansion and Th1 polarization of non-regulatory CD4+ T cells. Cell Immunol 231, 75-84. 33. Kim, J.W. et al. (2005) Chemokine $C$ receptor 7 expression and protection of circulating CD8+ T lymphocytes from apoptosis. Clin Cancer Res 11 (21), 7901-10. 34. Lopez-Cotarelo, P. et al. (2015) A novel MEK-ERK-AMPK signaling axis controls chemokine receptor CCR7-dependent survival in human mature dendritic cells. J Biol Chem 290, 827-840. 35. Morley, S.C. et al. (2010) The actin-bundling protein L-plastin dissociates CCR7 proximal signaling from CCR7-induced motility. J Immunol 184 (7), 3628-3638.

36. Ploix, C. et al. (2001) A ligand for the chemokine receptor CCR7 can influence the homeostatic proliferation of CD4 T cells and progression of autoimmunity. J Immunol 167, 6724-30.

661

662 37. Riol-Blanco, L. et al. (2005) The chemokine receptor CCR7 activates in dendritic cells two signaling modules that independently regulate chemotaxis and migratory speed. J Immunol $174,4070-80$.

38. Robertson, M.J. (2002) Role of chemokines in the biology of natural killer cells. J Leukoc Biol 71, 173-183.

39. Sanchez-Sanchez, N. et al. (2004) Chemokine receptor CCR7 induces intracellular signaling that inhibits apoptosis of mature dendritic cells. Blood 104, 619-25.

40. Stein, J.V. et al. (2000) The CC chemokine thymus-derived chemotactic agent 4 (TCA-4, secondary lymphoid tissue chemokine, 6Ckine, exodus-2) triggers lymphocyte functionassociated antigen 1-mediated arrest of rolling $T$ lymphocytes in peripheral lymph node high endothelial venules. J Exp Med 191 (1), 61-76.

41. Stein, J.V. et al. (2003) CCR7-mediated physiological lymphocyte homing involves activation of a tyrosine kinase pathway. Blood 101, 38-44.

42. Torres Bacete, J. et al. (2015) The Mammalian Sterile20-like 1 (Mst1) kinase controls selective CCR7-dependent functions in human dendritic cells. J. Immunol. 195, 973-81.

43. Yanagawa, Y. and Onoe, K. (2002) CCL19 induces rapid dendritic extension of murine dendritic cells. Blood 100, 1948-1956.

678 44. Yanagawa, Y. and Onoe, K. (2003) CCR7 ligands induce rapid endocytosis in mature 679 dendritic cells with concomitant up-regulation of Cdc42 and Rac activities. Blood 101, 4923-9.

680 45. Zhou, S. et al. (2010) SLC/CCR7 stimulates the proliferation of BMDCs by the pNF-kappaB p65 pathway. Anat Rec (Hoboken) 293, 48-54.

46. Link, A. et al. (2007) Fibroblastic reticular cells in lymph nodes regulate the homeostasis of naive T cells. Nat Immunol 8, 1255-1265.

47. Adachi, K. and Davis, M.M. (2011) T-cell receptor ligation induces distinct signaling pathways in naive vs antigen-experienced T cells. Proc Natl Acad Sci U S A 108, 1549-1554.

48. Ruckes, T. et al. (2001) Autocrine antiapoptotic stimulation of cultured adult T-cell leukemia cells by overexpression of the chemokine I-309. Blood 98, 1150-1159.

49. Islam, S.A. et al. (2013) Identification of human CCR8 as a CCL18 receptor. J Exp Med 210, 1889-1898.

50. Youn, B.S. et al. (2001) Blocking of c-FLIP(L)--independent cycloheximide-induced apoptosis or Fas-mediated apoptosis by the CC chemokine receptor 9/TECK interaction. Blood 98, 92533.

51. Crozat, K. et al. (2010) The XC chemokine receptor 1 is a conserved selective marker of mammalian cells homologous to mouse CD8alpha+ dendritic cells. J Exp Med 207, 1283-1292. 52. Hazeldine, J. et al. (2014) Impaired neutrophil extracellular trap formation: a novel defect in the innate immune system of aged individuals. Aging Cell 13, 690-698.

53. Pang, L. et al. (2013) Pseudogout-associated inflammatory calcium pyrophosphate dihydrate microcrystals induce formation of neutrophil extracellular traps. J Immunol 190, 6488-6500. 
54. Tharp, W.G. et al. (2006) Neutrophil chemorepulsion in defined interleukin-8 gradients in vitro and in vivo. J Leukoc Biol 79, 539-54.

55. Lippert, U. et al. (1998) Expression and functional activity of the IL-8 receptor type CXCR1 and CXCR2 on human mast cells. J Immunol 161 (5), 2600-8.

56. Walz, A. et al. (1991) [Ca2+]i changes and respiratory burst in human neutrophils and monocytes induced by NAP-1/interleukin-8, NAP-2, and gro/MGSA. J Leukoc Biol 50, 279-286. 57. Gibbons, D. et al. (2014) Interleukin-8 (CXCL8) production is a signatory $T$ cell effector function of human newborn infants. Nat Med 20, 1206-1210.

58. Proost, P. et al. (1993) Identification of a novel granulocyte chemotactic protein (GCP-2) from human tumor cells. In vitro and in vivo comparison with natural forms of GRO, IP-10, and IL-8. J Immunol 150, 1000-10.

59. Walz, A. et al. (1989) Effects of the neutrophil-activating peptide NAP-2, platelet basic protein, connective tissue-activating peptide III and platelet factor 4 on human neutrophils. J Exp Med 170, 1745-50.

60. Kredel, S. et al. (2014) Phenotypic analysis of chemokine-driven actin reorganization in primary human neutrophils. Assay Drug Dev Technol 12 (2), 120-128.

61. Proost, P. et al. (1993) Human and bovine granulocyte chemotactic protein-2: complete amino acid sequence and functional characterization as chemokines. Biochemistry 32, 1017010177.

62. Khajoee, V. et al. (2006) Novel roles of osteopontin and CXC chemokine ligand 7 in the defence against mycobacterial infection. Clin Exp Immunol 143, 260-268.

63. Walz, A. et al. (1991) Structure and neutrophil-activating properties of a novel inflammatory peptide (ENA-78) with homology to interleukin 8. J Exp Med 174, 1355-1362.

64. Green, S.P. et al. (1996) Interleukin-8 (IL-8), melanoma growth-stimulatory activity, and neutrophil-activating peptide selectively mediate priming of the neutrophil NADPH oxidase through the type A or type B IL-8 receptor. J Biol Chem 271 (41), 25400-5.

65. Rousselle, A. et al. (2013) CXCL5 limits macrophage foam cell formation in atherosclerosis. J Clin Invest 123, 1343-1347.

66. Smith, D.F. et al. (2005) GRO family chemokines are specialized for monocyte arrest from flow. Am J Physiol Heart Circ Physiol 289 (5), H1976-84.

67. Moser, B. et al. (1990) Neutrophil-activating properties of the melanoma growthstimulatory activity. J Exp Med 171, 1797-802.

68. Jin, L. et al. (2014) CXCL1 contributes to host defense in polymicrobial sepsis via modulating T cell and neutrophil functions. J Immunol 193, 3549-58.

69. von Hundelshausen, P. et al. (2007) Platelet-derived chemokines in vascular biology. Thromb Haemost 97 (5), 704-13.

70. Kohrgruber, N. et al. (2004) Plasmacytoid dendritic cell recruitment by immobilized CXCR3 ligands. J Immunol 173, 6592-602.

71. Whiting, D. et al. (2004) Chemokine monokine induced by IFN-gamma/CXC chemokine ligand 9 stimulates $\mathrm{T}$ lymphocyte proliferation and effector cytokine production. J Immunol $172,7417-24$.

72. Karin, N. et al. (2016) Biased signaling pathways via CXCR3 control the development and function of CD4+ T cell subsets. J Leukoc Biol 99, 857-862.

73. Groom, J.R. et al. (2012) CXCR3 chemokine receptor-ligand interactions in the lymph node optimize CD4+ T helper 1 cell differentiation. Immunity 37 (6), 1091-1103.

74. Zohar, Y. et al. (2014) CXCL11-dependent induction of FOXP3-negative regulatory T cells suppresses autoimmune encephalomyelitis. J Clin Invest 124, 2009-2022.

75. Shimaoka, T. et al. (2004) Cell surface-anchored SR-PSOX/CXC chemokine ligand 16 mediates firm adhesion of CXC chemokine receptor 6-expressing cells. J Leukoc Biol 75, 267274.

76. Geissmann, F. et al. (2005) Intravascular immune surveillance by CXCR6+ NKT cells patrolling liver sinusoids. PLoS Biol 3 (4), e113. 
77. Germanov, E. et al. (2008) Critical role for the chemokine receptor CXCR6 in homeostasis and activation of CD1d-restricted NKT cells. J Immunol 181, 81-91.

78. Braun, M.C. et al. (2000) Selective suppression of IL-12 production by chemoattractants. J Immunol 164, 3009-3017.

79. Gu, L. et al. (2000) Control of $\mathrm{TH} 2$ polarization by the chemokine monocyte chemoattractant protein-1. Nature 404, 407-411.

80. Jiang, Y. et al. (1992) Monocyte chemoattractant protein-1 regulates adhesion molecule expression and cytokine production in human monocytes. J Immunol 148, 2423-2428.

81. Sierra-Filardi, E. et al. (2014) CCL2 shapes macrophage polarization by GM-CSF and M-CSF: identification of CCL2/CCR2-dependent gene expression profile. J Immunol 192 (8), 3858-67.

82. Menten, P. et al. (2002) Macrophage inflammatory protein-1. Cytokine Growth Factor Rev $13,455-481$.

83. Diaz-Guerra, E. et al. (2007) CCL2 inhibits the apoptosis program induced by growth factor deprivation, rescuing functional T cells. J Immunol 179 (11), 7352-7.

84. Karpus, W.J. et al. (1997) Differential CC chemokine-induced enhancement of T helper cell cytokine production. J Immunol 158, 4129-4136.

85. Chensue, S.W. et al. (1996) Role of monocyte chemoattractant protein-1 (MCP-1) in Th1 (mycobacterial) and Th2 (schistosomal) antigen-induced granuloma formation: relationship to local inflammation, Th cell expression, and IL-12 production. J Immunol 157, 4602-8.

86. Aliberti, J. et al. (2000) CCR5 provides a signal for microbial induced production of IL-12 by CD8 alpha+ dendritic cells. Nat Immunol 1, 83-87.

87. Zou, W. et al. (2000) Macrophage-derived dendritic cells have strong Th1-polarizing potential mediated by beta-chemokines rather than IL-12. J Immunol 165, 4388-96.

88. Trifilo, M.J. and Lane, T.E. (2004) The CC chemokine ligand 3 regulates $C D 11 c+C D 11 b+C D 8$ alpha- dendritic cell maturation and activation following viral infection of the central nervous system: implications for a role in T cell activation. Virology 327, 8-15.

89. Locati, M. et al. (2002) Analysis of the gene expression profile activated by the CC chemokine ligand 5/RANTES and by lipopolysaccharide in human monocytes. J Immunol 168, 3557-3562.

90. Chabot, V. et al. (2006) CCL5-enhanced human immature dendritic cell migration through the basement membrane in vitro depends on matrix metalloproteinase-9. J Leukoc Biol 79, 767-778.

91. Kim, C.S. et al. (2011) Potential involvement of CCL23 in atherosclerotic lesion formation/progression by the enhancement of chemotaxis, adhesion molecule expression, and MMP-2 release from monocytes. Inflamm Res 60, 889-95.

92. Kim, J. et al. (2010) CKbeta8/CCL23 and its isoform CKbeta8-1 induce up-regulation of cyclins via the $\mathrm{G}(\mathrm{i}) / \mathrm{G}(\mathrm{o})$ protein/PLC/PKCdelta/ERK leading to cell-cycle progression. Cytokine 50, 42-9.

93. Kim, J. et al. (2010) CK beta 8/CCL23 induces cell migration via the Gi/Go protein/PLC/PKC delta/NF-kappa B and is involved in inflammatory responses. Life Sci 86, 300-308.

94. Nardelli, B. et al. (1999) Characterization of the signal transduction pathway activated in human monocytes and dendritic cells by MPIF-1, a specific ligand for CC chemokine receptor 1. $\mathrm{J}$ Immunol 162, 435-44.

95. Cappello, P. et al. (2006) CC-chemokine ligand 16 induces a novel maturation program in human immature monocyte-derived dendritic cells. J Immunol 177, 6143-6151.

96. Ogilvie, P. et al. (2003) Eotaxin-3 is a natural antagonist for CCR2 and exerts a repulsive effect on human monocytes. Blood 102, 789-794.

97. Badewa, A.P. et al. (2002) Regulatory effects of eotaxin, eotaxin-2, and eotaxin-3 on eosinophil degranulation and superoxide anion generation. Exp Biol Med (Maywood) 227, 645651.

98. Elsner, J. et al. (1996) Human eotaxin represents a potent activator of the respiratory burst of human eosinophils. Eur J Immunol 26, 1919-1925. 
99. Ness, T.L. et al. (2006) CCR4 is a key modulator of innate immune responses. J Immunol 177, 7531-7539.

100. Matsukawa, A. et al. (2000) Pivotal role of the CC chemokine, macrophage-derived chemokine, in the innate immune response. J Immunol 164, 5362-5368.

101. Dichmann, S. et al. (2001) Fractalkine induces chemotaxis and actin polymerization in human dendritic cells. Inflamm Res 50, 529-533.

102. Boehme, S.A. et al. (2000) The chemokine fractalkine inhibits Fas-mediated cell death of brain microglia. J Immunol 165, 397-403.

103. Pallandre, J.R. et al. (2008) Dendritic cell and natural killer cell cross-talk: a pivotal role of CX3CL1 in NK cytoskeleton organization and activation. Blood 112, 4420-4424.

104. Karlmark, K.R. et al. (2010) The fractalkine receptor CX(3)CR1 protects against liver fibrosis by controlling differentiation and survival of infiltrating hepatic monocytes. Hepatology 52, 1769-82.

105. Landsman, L. et al. (2009) CX3CR1 is required for monocyte homeostasis and atherogenesis by promoting cell survival. Blood 113 (4), 963-72.

106. Limatola, C. and Ransohoff, R.M. (2014) Modulating neurotoxicity through CX3CL1/CX3CR1 signaling. Front Cell Neurosci 8, 229.

107. Byrnes, H.D. et al. (1999) Macrophage inflammatory protein-3 beta enhances IL-10 production by activated human peripheral blood monocytes and T cells. J Immunol 163, 471520.

108. Pease, J.E. and Williams, T.J. (2001) Eotaxin and asthma. Curr Opin Pharmacol 1, 248-253. 109. Ogawa, H. et al. (2004) Regulated production of the chemokine CCL28 in human colon epithelium. Am J Physiol Gastrointest Liver Physiol 287, G1062-9.

110. White, G.E. et al. (2013) CC chemokine receptors and chronic inflammation--therapeutic opportunities and pharmacological challenges. Pharmacol Rev 65, 47-89.

111. Moser, B. and Loetscher, P. (2001) Lymphocyte traffic control by chemokines. Nat Immunol 2, 123-128.

112. Vanbervliet, B. et al. (2003) The inducible CXCR3 ligands control plasmacytoid dendritic cell responsiveness to the constitutive chemokine stromal cell-derived factor 1 (SDF1)/CXCL12. J Exp Med 198, 823-30.

113. Umehara, H. et al. (2004) Fractalkine in vascular biology: from basic research to clinical disease. Arterioscler Thromb Vasc Biol 24, 34-40.

114. Wendt, E. and Keshav, S. (2015) CCR9 antagonism: potential in the treatment of inflammatory bowel disease. Clin. Exp. Gastroenterol. 8, 119-130.

115. Matloubian, M. et al. (2000) A transmembrane CXC chemokine is a ligand for HIVcoreceptor Bonzo. Nat Immunol 1, 298-304.

116. Dorner, B.G. et al. (2009) Selective expression of the chemokine receptor XCR1 on crosspresenting dendritic cells determines cooperation with CD8+ T cells. Immunity 31 (5), 823-833. 117. Fox, J.C. et al. (2015) Structural and agonist properties of XCL2, the other member of the C-chemokine subfamily. Cytokine 71, 302-311.

118. Balabanian, K. et al. (2003) Role of the chemokine stromal cell-derived factor 1 in autoantibody production and nephritis in murine lupus. J Immunol 170, 3392-400.

119. Rangel-Moreno, J. et al. (2007) Pulmonary expression of CXC chemokine ligand 13, CC chemokine ligand 19, and CC chemokine ligand 21 is essential for local immunity to influenza. Proc Natl Acad Sci U S A 104, 10577-10582.

120. Svensson, M. and Agace, W.W. (2006) Role of CCL25/CCR9 in immune homeostasis and disease. Expert Rev Clin Immunol 2, 759-773.

121. Gombert, M. et al. (2005) CCL1-CCR8 interactions: an axis mediating the recruitment of T cells and Langerhans-type dendritic cells to sites of atopic skin inflammation. J Immunol 174 (8), 5082-91.

122. Taub, D.D. et al. (1995) Alpha and beta chemokines induce NK cell migration and enhance NK-mediated cytolysis. J Immunol 155 (8), 3877-3888. 
123. Mellado, M. et al. (2001) Chemokine signaling and functional responses: the role of receptor dimerization and TK pathway activation. Annu Rev Immunol 19, 397-421.

858 124. Neptune, E.R. and Bourne, H.R. (1997) Receptors induce chemotaxis by releasing the 859 betagamma subunit of $\mathrm{Gi}$, not by activating Gq or Gs. Proc Natl Acad Sci U S A 94, 1448986014494.

861 125. Wong, M.M. and Fish, E.N. (2003) Chemokines: attractive mediators of the immune response. Semin Immunol 15 (1), 5-14. 126. Yang, Y.M. et al. (2001) Beta-chemokine induction of activation protein-1 and cyclic AMP responsive element activation in human myeloid cells. Cell Growth Differ 12, 211-221. 127. Sallusto, F. et al. (2000) The role of chemokine receptors in primary, effector, and memory immune responses. Annu Rev Immunol 18, 593-620. 128. Marsland, B.J. et al. (2005) CCL19 and CCL21 induce a potent proinflammatory differentiation program in licensed dendritic cells. Immunity 22, 493-505. 129. Braun, A. et al. (2011) Afferent lymph-derived T cells and DCs use different chemokine receptor CCR7-dependent routes for entry into the lymph node and intranodal migration. Nat Immunol 12, 879-887.

130. Platt, C.D. et al. (2010) Mature dendritic cells use endocytic receptors to capture and present antigens. Proc Natl Acad Sci U S A 107, 4287-4292.

131. Gerszten, R.E. et al. (1999) MCP-1 and IL-8 trigger firm adhesion of monocytes to vascular endothelium under flow conditions. Nature 398 (6729), 718-723.

132. Varona, R. et al. (2001) CCR6-deficient mice have impaired leukocyte homeostasis and altered contact hypersensitivity and delayed-type hypersensitivity responses. J Clin Invest 107, R37-R45.

879 133. Forster, R. et al. (1999) CCR7 coordinates the primary immune response by establishing functional microenvironments in secondary lymphoid organs. Cell 99 (1), 23-33. 134. Forster, R. et al. (1996) A putative chemokine receptor, BLR1, directs B cell migration to defined lymphoid organs and specific anatomic compartments of the spleen. Cell 87, 10371047.

884 135. Nie, Y. et al. (2004) The role of CXCR4 in maintaining peripheral B cell compartments and 885 humoral immunity. J Exp Med 200, 1145-1156.

886 136. Leon, B. et al. (2012) Regulation of $T(H) 2$ development by CXCR5+ dendritic cells and 887 lymphotoxin-expressing B cells. Nat Immunol 13 (7), 681-690.

888 137. Charo, I.F. and Ransohoff, R.M. (2006) The many roles of chemokines and chemokine 889 receptors in inflammation. N Engl J Med 354 (6), 610-621.

890 138. Bardi, G. et al. (2001) The T cell chemokine receptor CCR7 is internalized on stimulation 891 with ELC, but not with SLC. Eur J Immunol 31 (11), 3291-7. 